\title{
Carcinoma de uraco, una neoplasia maligna poco frecuente
}

\author{
Natalia García Salazar*, Margarita María Pérez Silva†, Marylin Acuña Hernández
}

\section{Resumen}

El carcinoma de uraco es una neoplasia maligna poco frecuente de predominio masculino entre la quinta a sexta década de la vida. Se localizan típicamente en la cúpula de la vejiga, con patrón histopatológico predominante de adenocarcinoma en el $90 \%$ de los casos. Tiene un curso indolente con manifestaciones clínicas en etapas tardías caracterizadas por hematuria, dolor abdominal, disuria, mucosuria, entre otros. El diagnóstico se basa en el hallazgo de la lesión por métodos endoscópicos e imagenológicos. Dado lo infrecuente de esta patología, se describe el caso de una paciente en su cuarta década de vida con infección de vías urinarias recurrente, en quien se documentó por medio de urografía por tomografía computarizada multicorte (UROTAC) de pólipo vesical resecado por resección transuretral (RTU) con reporte histológico de adenocarcinoma de tipo entérico.

Palabras clave: CARCINOMA DE URACO PRUEBAS DIAGNÓSTICAS DE RUTINA

Key words: $\quad$ URACHAL CARCINOMA ROUTINE DIAGNOSTIC TESTS

\section{Introducción}

El carcinoma de uraco es una neoplasia maligna que corresponde al $0,7 \%$ de todos los cánceres de vejiga y al $35 \%$ de adenocarcinomas vesicales ${ }^{(1)}$. Es más frecuente en hombres con una relación $5: 1^{(2,15)}$, de predominio entre la quinta a sexta década de la vida ${ }^{(3)}$.

En cuanto a su origen, el uraco es una estructura de 5 a $10 \mathrm{~cm}$ de longitud proveniente de la alantoides, que pasa desde la cúpula vesical hasta el ombligo y se convierte en un cordón fibroso obliterado en el adulto ${ }^{(4)}$. Se constituye de tres capas: una externa constituida por músculo liso, una intermedia constituida por tejido conectivo submucosa y una interna de tipo luminal. De las anteriores, las células epiteliales que constituyen cada capa dan lugar al carcinoma de uraco $^{(5)}$.

\section{Caso clínico}

Paciente mujer en su cuarta década de vida, presenta dos años con síntomas urinarios irritativos y mucosuria con infección de vías urinarias recurrentes, se indicó tratamiento antibiótico guiado por urocultivo. Se realizó UROTAC con hallazgo de lesión polipoide en vejiga de $22 \mathrm{~mm}$. Posteriormente es llevada a cistoscopía, documentando hallazgo anterior en cúpula vesical, la cual es removida a través de RTU con reporte de histopatología de adenocarcinoma de tipo entérico.

Se llevó a junta de oncología donde se conceptuó manejo para carcinoma de uraco a través de cirugía, por lo que se realizó cistectomía parcial más resección de seno uracal y linfadenectomía radical pélvica. Dos meses posteriores a la intervención quirúrgica, presentó dolor severo suprapúbico e inguinal, con sospecha de recaída

\footnotetext{
* Médica. Servicio clínica del dolor y cuidado paliativo, Grupo ALIVIAR, Bucaramanga, Santander, Colombia.

† Médica en Servicio Social Obligatorio, Fundación Oftalmológica de Santander - Clínica Carlos Ardila Lülle (FOSCAL) Bucaramanga, Santander, Colombia.

‡ Especialista en Epidemiologia. Universidad Autónoma de Bucaramanga (UNAB) Bucaramanga, Santander, Colombia.

Correspondencia: Marylin Acuña Hernández. Correo electrónico: Nandu152@hotmail.com

Recibido: 9/11/17

Aceptado: 16/1/18
} 
Tabla 1. Criterios M.D. Anderson Cancer Center.

Localización en el domo vesical o en la línea media de la vejiga

Clara delimitación del tumor y el urotelio sano

Criterios que apoyan el diagnóstico:

Histología de origen entérico

Ausencia de displasia urotelial

Ausencia de cistitis quística o cistitis

Ausencia de adenocarcinoma primario en algún otro órgano

Fuente: Cruz et al ${ }^{(7)}$.

se realizó antígeno carcinoembrionario, el cual estaba elevado, y se complementó búsqueda con 18 F-FDG PET-CT (tomografía por emisión de positrones) con evidencia de lesiones hipermetabólicas en pelvis, vejiga y cuello de la vejiga y resonancia nuclear magnética (RNM) contrastada de pelvis (figura 1), con masa prevesical isointensa en T1 y T2, además de nódulo paravesical derecho entre el paquete vascular ilíaco externo derecho y el receso lateral derecho de la vejiga, el cual es isointenso en T1 y T2 de $8 \mathrm{~mm}$ de diámetro. Se realiza diagnóstico de recaída locorregional, realizándose nuevo abordaje quirúrgico y terapia coadyuvante con quimioterapia y adecuada evolución clínica durante seguimiento.

\section{Discusión}

El carcinoma de uraco se localiza típicamente en la cúpula de la vejiga ${ }^{(4)}$. Morfológicamente, el $90 \%$ son adenocarcinomas ${ }^{(4)}$, presentando cinco subtipos histopatológicos: células intestinales (50\%), mucinoso, en anillo de sello, mixto y adenocarcinoma no especificado ${ }^{(5)}$.

Clínicamente se manifiesta con hematuria $(73 \%)$, dolor abdominal (14\%), disuria (13\%), mucosuria $(10 \%)^{(3)}$, síntomas irritativos $(40 \%)$, masa palpable en la parte baja del abdomen (17\%), bacteriuria (8\%), flujo mucoso umbilical $(2 \%)^{(7)}$.

El diagnóstico actualmente se realiza empleando los criterios M.D. Anderson Cancer Center ${ }^{(6)}$ (tabla 1). La cistoscopía es la herramienta diagnóstica más importante dado que ubica la lesión al interior de la vejiga.

Las imágenes forman parte importante para el diagnóstico y estadificación de la enfermedad. La tomografía computada (TC) es la imagen más empleada, reportando hallazgos característicos como la presencia de masa supravesical anterior a la línea media y calcificaciones periféricas $^{(5)}$. La RNM permite ampliar el compromiso tumoral al encontrarse una masa sólida o quísti-
Tabla 2. Estadificación de Sheldon et al, para carcinoma del uraco.

- Estadio l: Tumor ubicado en la mucosa del uraco

- Estadio II: Invasión confinada al uraco

- Estadio Illa: Extensión local a la vejiga

- Estadio Illb: Extensión a la pared abdominal

- Estadio IIIc: Invasión al peritoneo

- Estadio IIId: Extensión a otra víscera diferente a la vejiga

- Estadio IVa: Invasión a ganglios linfáticos regionales

- Estadio IVb: Metástasis a distancia.

Fuente: Sheldon et al ${ }^{(11)}$.

ca heterogénea en el trayecto del uraco con calcificaciones y ganglios linfáticos aumentados de tamaño ${ }^{(7)}$.

En la actualidad se emplean técnicas de medicina nuclear para determinar la extensión de la enfermedad, como lo es la 18 F-FDG PET-CT. Zeman y colaboradores reportan que estos tumores muestran una variación considerable en su grado de captación dependiendo de las características histopatológicas: los tumores mucinosos dada su baja celularidad tienen baja avidez por la 18 F-FDG en contraste con la variante intestinal que presenta alta avidez ${ }^{(8)}$.

$\mathrm{Al}$ igual que las imágenes diagnósticas y endoscópicas, los marcadores tumorales han demostrado ser útiles en el diagnóstico y seguimiento de la enfermedad usando el antígeno carcinoembrionario, antígeno Ca19-9 y antígeno Ca-125, los cuales suelen estar elevados en caso de enfermedad activa ${ }^{(9)}$.

La estadificación inicial se realiza con la clasificación de Sheldon y colaboradores ${ }^{(10)}(\text { tabla } 2)^{(6)}$.

El gold standard para tratamiento en enfermedad no metastásica en la actualidad es la cirugía, que incluye una amplia escisión local del uraco con una cistectomía parcial, resección umbilical y del tejido blando circundante combinado con la linfadenectomía pélvica bilate$\mathrm{ral}^{(3)}$. Este manejo proporciona una supervivencia libre de enfermedad a largo plazo ${ }^{(3,11)}$. En la actualidad la cistectomía radical se considera en caso de recurrencia o metástasis, dado que ha mostrado mejoría de la supervivencia en algunos casos reportados ${ }^{(4)}$.

Dado el curso indolente de esta enfermedad, la mayoría de los diagnósticos suele realizarse en etapas avanzadas de la enfermedad con metástasis a distancia.

La recurrencia en el primer año es muy frecuente (alrededor de $54 \%)^{(3)}$, siendo los sitios más comunes la pelvis, vejiga, incisión quirúrgica y pared abdominal ${ }^{(12)}$, 

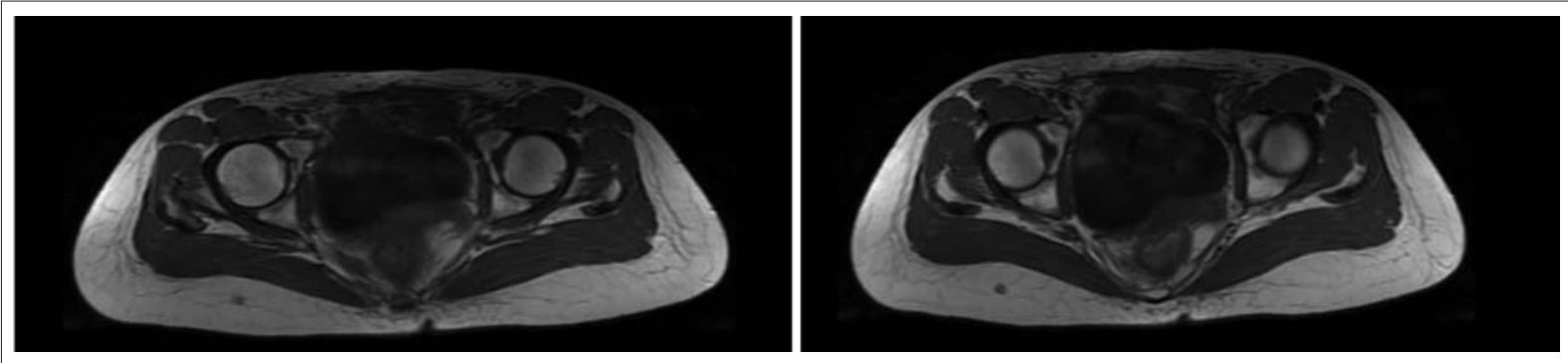

Figura 1. Imagen de resonancia magnética que evidencia masa prevesical con extenso compromiso pélvico (corte axial).

siendo factores de riesgo la presencia de márgenes positivos en la cirugía, compromiso de ganglios linfáticos regionales y ausencia en la resección del ombligo ${ }^{(13)}$. Los órganos más frecuentemente afectados por metástasis son el pulmón (50\%), ganglios linfáticos regionales (46\%), hueso de predominio columna vertebral (30\%), intestino $(30 \%)$, cerebro $(20 \%)$ e hígado $(16 \%)^{(3,12)}$.

En estos casos no existe un manejo estándar, por lo que se refiere en la literatura el uso de quimioterapia y radioterapia como terapia coadyuvante ${ }^{(13)}$, pero a cinco años la supervivencia en este tipo de paciente no supera el $20 \%^{(12,14)}$.

\section{Conclusión}

El carcinoma de uraco es una patología poco frecuente de curso indolente que suele diagnosticarse en estadios avanzados de la enfermedad. Para el manejo inicial se indica una resección quirúrgica amplia del bloque tumoral con alto riesgo de recurrencia y metástasis a distancia en el primer año, lo que disminuye la supervivencia a cinco años, por lo que se emplean métodos coadyuvantes como la radioterapia y quimioterapia para mejorar la calidad de vida.

\section{Abstract}

Urachal carcinoma is a rare malignant neoplasm of male predominance between the fifth to sixth decade of life. It is typically located in the dome of the bladder, with a predominant histopathological pattern of adenocarcinoma in $90 \%$ of cases. It has an indolent course with clinical manifestations in late stages characterized by hematuria, abdominal pain, dysuria, mucosuria, among others. Diagnosis is based on the finding of the lesion by endoscopic and imaging techniques. Given the infrequent nature of this pathology, we describe the case of a female patient in her fourth decade of life with recurrent urinary tract infection, who was documented by MSCTU of bladder polyp resected by TURP with a histological report of enteric-type adenocarcinoma.

\section{Resumo}

O carcinoma de úraco, é uma neoplasia maligna pouco frequente, predominantemente masculina que se manifesta entre a quinta e a sexta década de vida. Sua localização típica é a cúpula vesical e em $90 \%$ dos casos sua histologia corresponde a adenocarcinomas. Apresenta evolução indolente com manifestações clínicas nas etapas tardias caracterizadas por hematúria, dor abdominal, disúria, mucosúria, entre outros. O diagnóstico está baseado no achado da lesão por métodos endoscópicos e de imagens. Considerando a baixa frequência desta patologia, descreve-se o caso de uma paciente de sexo feminino em sua quarta década de vida com infecção recorrente das vias urinarias, na qual foi registrado, através de UROTAC, um pólipo vesical ressecado por RTU com laudo histológico de adenocarcinoma de tipo entérico.

\section{Bibliografía}

1. Reuter $\mathbf{V}$. The Urothelial tract: Renal pelvis, ureter, urinary bladder and urethra. Philadelphia: Lippincott Williams and Wilkins; 2004. p. 2062-3.

2. Gopalan A, Sharp DS, Fine SW. Urachal carcinoma: a clinicopathologic analysis of 24 cases with outcome correlation. Am J Surg Pathol. 2009;33: 659 - 68.

3. Szarvas T, Módos O, Niedworok C, Reis H, Szendröi A, Szász M. Clinical, prognostic, and therapeutic aspects of urachal carcinoma - A comprehensive review with meta-analysis of 1,010 cases. Urologic Oncology: Seminars and Original Investigations. 2016; 34: 388-98.

4. Behrendt M, Jong J, Rhijn V. Urachal cancer: contemporary review of the pathological, surgical, and prognostic aspects of this rare disease. Minerva Urol Nefrol. 2016; 68(2): 172-84.

5. Quan J, Pan X, Jin L, He T, Hu J, Shi B, et al. Urachal carcinoma: Report of two cases and review of the literatura. Molecular and clinical oncology. 2017; 6: 101-4.

6. Cruz P, Ramírez M, Iborra I, Rubio J, Pinto F, Gómez-Ferrer A, et al. Adenocarcinoma de uraco: ¿qué tanto sabemos? Revista Mexicana de Urología. 2014; 74: 301-7. 
7. Jung H, Sun J, Park S, Kwon Y, Lim H. Treatment outcome and relevance of palliative chemotherapy in urachal cancer. Chemotherapy. 2014; 60: 73-80.

8. Zeman M, Silver E, Akin E. CT and PET Findings for Urachal Adenocarcinoma: A Case Report. Annals of Clinical. 2017; 2: 1252.

9. Hayashi T, Yuasa T, Uehara S, et al. Clinical outcome of urachal cancer in Japanese patients. Int J Clin Oncol 2016; 21:133-8.

10. Sheldon C, Clayman R, González R, Williams R, Fraley E. Malignant urachal lesions. J Urol 1984; 131: 1-8.

11. Kumar N, Khosla D, Kumar R, Mandal A, Saikia U, Kapoor R, Shrawan K. et al. Urachal carcinoma: Clinicopatho- logical features, treatment and outcome. Journal of Cancer Research and Therapeutics 2014; July-September: 10 - 3.

12. Ashley R, Inman B, Sebo T, Leibovich B, Blute M, Kwon E, et al. Urachal carcinoma: Clinicopathologic features and long-term outcomes of an aggressive malignancy. Cancer. 2006; 107:712-20.

13. Pinthus J, Haddad R, Trachtenberg J, Holowaty E, Bowler J, Herzenberg A, et al. Population based survival data on urachal tumors. J Urol. 2006;1 75:2042-7; discussion 2047.

14. Niedworok C, Panitz M, Szarvas T, et al. Urachal carcinoma of the bladder - impact of clinical and immunohistochemical parameters on patients' prognosis. J Urol. 2015; 10 : 1016. 